\title{
Vancomycin in the treatment of adult intra- abdominal infections: do we have strong evidences?
}

This article was published in the following Dove Press journal: Infection and Drug Resistance

\author{
Song Liu \\ Meng Wang \\ Wenxian Guan \\ Department of General Surgery, \\ Nanjing Drum Tower Hospital, \\ The Affiliated Hospital of Nanjing \\ University Medical School, Nanjing \\ 210008, China
}

Correspondence: Meng Wang; Wenxian Guan

Department of General Surgery, Nanjing Drum Tower Hospital, The Affiliated Hospital of Nanjing University Medical School, 32I Zhongshan Road, Nanjing, 210008 , China

Tel 86256818222260931 ;

86256818222260999

Email wangmeng00I@263.net; guan_wenxian@sina.com

\begin{abstract}
The management of intra-abdominal infections (IAIs) primarily includes adequate antimicrobial therapy and appropriate source control. Vancomycin is a fundamental and most effective antimicrobial agent. The aim of this study is to search and evaluate the quality of clinical evidences regarding the exclusive use of vancomycin for the management of adult IAIs. For this purpose, we first summarized the recommendations on exclusive use of vancomycin in adult IAIs from six leading guidelines and excavated the relevant supporting references. We subsequently conducted a literature search to screen eligible clinical studies in this field for possible systematic review. Our investigation demonstrates that the exclusive use of vancomycin is recommended in restricted indications, that is, IAIs caused by Enterococcus spp. or methicillin-resistant Staphylococcus aureus. However, the supporting references in the guidelines are more subjective instead of objective, which mainly originate from experts' personal experiences or from the therapeutic efficacy of vancomycin in other types of infections rather than in IAIs. Furthermore, our literature search fails to find high-level evidence. In conclusion, current low-level evidences are inadequate to elicit strong recommendations on the exclusive use of vancomycin in the treatment of adult IAIs. Our study would be helpful for the rational use of vancomycin and deceleration of the emerging vancomycin resistance rates.
\end{abstract}

Keywords: intra-abdominal infection, vancomycin, guideline, anti-infective agents

\section{Background}

Intra-abdominal infections (IAIs) represent a variety of conditions that involve pathologic inflammation of intra-abdominal organs or peritoneum. ${ }^{1}$ IAIs are mainly classified into two types: uncomplicated IAIs and complicated IAIs. Uncomplicated IAIs are defined as an infectious process only limited within single organ and does not involve peritoneum. In contrast, in complicated IAIs, the infection extends beyond the organ and causes either localized or diffuse peritonitis. ${ }^{2}$

For both uncomplicated and complicated IAIs, antimicrobials and source control are two fundamental strategies. In recent years, the increasing disease burden and resistance rate of microorganisms are in parallel with the emergence of new antibiotics and new source control approaches. ${ }^{3}$ In response to the arising challenges, academic societies have released individual guidelines on the rational use of antibiotics in the management of IAIs.

Vancomycin is generally considered as the last resort of antibiotics for the treatment of Gram-positive bacteria in serious and life-threatening infections. ${ }^{4,5}$ Because of its high efficacy toward Gram-positive microorganisms, the total usage increased from small quantities to over $14,000 \mathrm{~kg}$ within two decades in Western countries. ${ }^{6}$ 
Nevertheless, vancomycin is currently confronting major challenges, including vancomycin-resistant Enterococci, ${ }^{7}$ vancomycin-tolerant Streptococcus pneumoniae, ${ }^{8}$ and vancomycin-resistant Staphylococcus aureus. ${ }^{9}$

To decelerate the emerging vancomycin resistance rate, current guidelines restrict the indications of vancomycin. In the current study, we first overviewed guideline recommendations on the exclusive use of vancomycin in the management of adult IAIs, with particular evaluation on the quality of supporting evidences. We subsequently performed a literature search to screen eligible studies in this field for possible systematic review.

\section{What do international guidelines say?}

We have reviewed the following six most recent guidelines or consensus released by a variety of geographically diversified professional societies in the management of IAIs: 1) Surgical Infection Society, version 2017 (SIS 2017), ${ }^{10} 2$ ) World Society of Emergency Surgery, version 2017 (WSES 2017), ${ }^{11}$ 3) Global Alliance, version 2016 (AGORA 2016), ${ }^{12} 4$ ) French National Guideline, version 2015 (France 2015), ${ }^{13}$ 5) Asian Perspective, version 2014 (Asia 2014), ${ }^{14}$ and 6) Infectious Diseases Society of America, version 2010 (IDSA 2010) ${ }^{15}$ (Table 1).

SIS 2017 is the fourth edition of IAIs management guideline following 1992, 2002, and 2010 versions released by the SIS. ${ }^{10}$ In this latest guideline, vancomycin is primarily suggested as an adjuvant drug in certain scenarios in the treatment of IAIs. For instance, "consider use of aztreonam plus metronidazole plus vancomycin for empiric therapy for higher-risk IAIs patients, especially for those with serious $\beta$-lactam allergies", "consider use of added ampicillin or vancomycin for empiric anti-enterococcal treatment in higher-risk patients if they are not being treated with piperacillin-tazobactam or imipenem-cilastatin". There are only two scenarios in which exclusive vancomycin is recommended, that is, consider use of vancomycin or teicoplanin for empiric therapy of hospital-acquired IAIs in patients at risk for infection from Enterococcus spp. or for empiric therapy of patients known to be colonized or at high risk for infection with methicillin-resistant Staphylococcus aureus (MRSA). Nevertheless, the panel has also explained in the text that the efficacy of vancomycin in the treatment of either Enterococcus or intra-abdominal MRSA infections has not been definitively evaluated. There is no available publication to provide objective clinical evidence. The above recommendations mainly rely on experts' personal experience or data from vancomycin in treating other types of MRSA infections (eg, skin/skin structure infection and urinary tract infection).

WSES 2017 is an update of the WSES 2013 guideline for the management of IAIs. ${ }^{11}$ In this guideline, vancomycin is mentioned as an adjuvant antibiotic to ceftazidime/ avibactam and metronidazole in critically ill patients with hospital-acquired IAIs. Vancomycin is also suggested in the management of Enterococcus spp. infection (especially ampicillin-resistant Enterococcus), but supporting evidences are not provided or discussed. We assume that the panel failed

Table I Recommendations on exclusive use of vancomycin in the management of intra-abdominal infections (IAls) in current guidelines

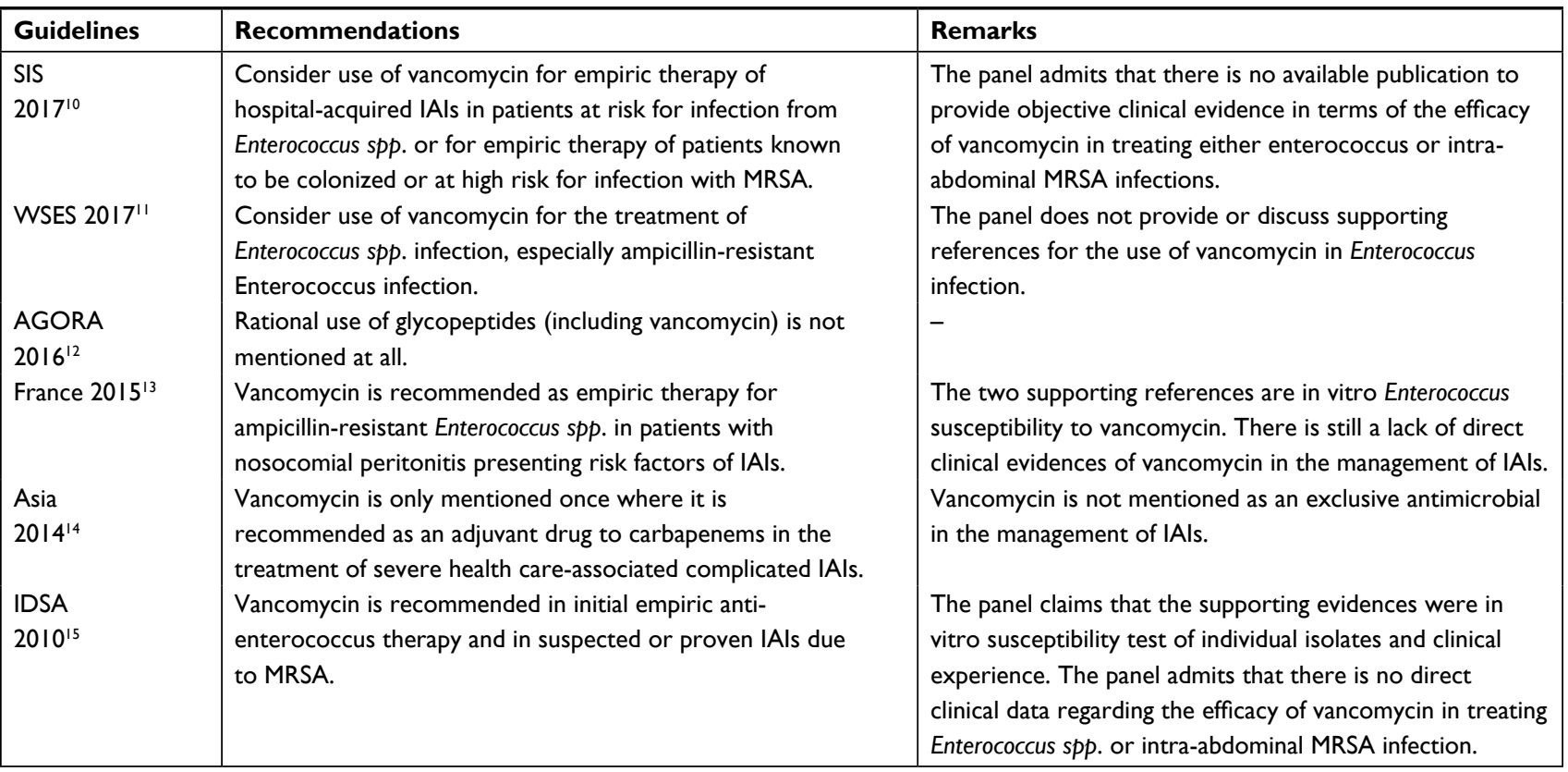

Abbreviation: MRSA, methicillin-resistant Staphylococcus aureus. 
to obtain published data in supporting the use of vancomycin in Enterococcus infection and referred parallel guidelines.

AGORA 2016 study is short for "a global alliance for optimizing antimicrobials rational use in IAIs". ${ }^{12}$ This study is carried out by an international task force from 79 countries and has illustrated the global burden of antimicrobial resistance based on shared regional epidemiological data. This study has also provided suggestions about rational use of each type of antimicrobials for IAIs patients. However, glycopeptides (including vancomycin and teicoplanin) are not mentioned at all in their statements.

France 2015 refers to the French National Guideline conducted jointly by the French Society of Anethesia and Intensive Care Medicine, French Intensive Care Society, French Infectious Diseases Society, and French Society of Digestive Surgery. ${ }^{13}$ Exclusive vancomycin is recommended as empiric therapy for ampicillin-resistant Enterococcus spp. in patients with nosocomial peritonitis presenting risk factors of IAIs. However, after tracking the two supporting references in the text, ${ }^{16,17}$ we conclude that this statement is based on in vitro data of anti-enterococcus activities of vancomycin, instead of clinical comparative cohort study. Therefore, there is still a lack of direct clinical evidences of vancomycin in the management of IAIs.

Asia 2014 is an Asian perspective in antibiotic management of complicated IAIs in adults. ${ }^{14}$ Based on Asian epidemiological data and resistance profile, the taskforce develops recommendations for antibiotic management of complicated IAIs in Asia. In this perspective, vancomycin is only mentioned once where it is recommended as an adjuvant drug to carbapenems in the treatment of severe health careassociated complicated IAIs. Vancomycin is not mentioned as an exclusive antimicrobial in the management of IAIs.

IDSA 2010 is a joint guideline for the diagnosis and management of complicated IAIs by the SIS and the IDSA. ${ }^{15}$ Similar to SIS 2017, WSES 2017, and France 2015, the IDSA 2010 recommends vancomycin in initial empiric antienterococcus therapy and in suspected or proven IAIs due to MRSA. Nevertheless, the panel has discussed in the text that their recommendation regarding the use of vancomycin in Enterococcus spp. was based on in vitro susceptibility test of individual isolates, and their recommendation regarding the use of vancomycin in MRSA was due to "vancomycin has been generally used to treat MRSA infection". They admit that there is no specific data regarding the efficacy of antibiotics in treating intra-abdominal MRSA infection.

\section{Literature search}

We conducted a computerized bibliographic search for all retrospective or prospective observational and/or experi- mental randomized studies as well as meta-analysis in the field of vancomycin and IAIs (including both uncomplicated and complicated IAIs). We included MEDLINE, Embase, Cochrane Library, and ClinicalTrials.gov databases up to May 2017 for the search of publications and ongoing trials with language restriction of English. The exclusion criteria were peritoneal dialysis study, animal study, epidemiology study, nonadult (pediatrics) study, nonoriginal publication (review, editorial), and informal publication (conference abstract). The following keywords and MeSH terms were used: "intra abdominal infection", "intraabdominal infection", and "vancomycin". We conducted an additional manual retrieval of relevant original references from current guidelines.

Figure 1 demonstrates the flowchart of eligible studies. A total of 1,409 references were identified, of which 685 were subsequently screened after removal of duplicates. Afterward, irrelevant pharmacodynamics studies $(n=208)$, animal studies $(n=141)$, epidemiology studies $(n=136)$, and pediatric studies $(n=49)$ were excluded. Irrelevant dissertation $(n=48)$, review/editorial $(n=31)$, case report $(n=26)$, and conference abstract $(n=17)$ were excluded as well. The remaining 29 articles together with 25 additional articles by manual retrieval from current guidelines were assessed for eligibility. After thorough full-text evaluation, 19 combination therapy (nonexclusive vancomycin) studies, 11 non-IAI-specific (IAI data unavailable) studies, 10 descriptive single-arm (noncomparative) studies, 9 in vitro/ex vivo studies, and 5 pharmacokinetics and/or pharmacodynamics and/or side-effect studies were excluded. Eventually, no eligible study remained for systematic review (Figure 1).

\section{Perspectives}

To summarize, the above six guidelines consistently recommend the exclusive use of vancomycin in two indications: IAIs caused by Enterococcus spp. or IAIs related to MRSA, especially in patients with drug-resistant Enterococcus spp. and/or severe hospital-acquired IAI.

Experimental susceptibility tests demonstrated that Staphylococcus aureus isolated from pus of severe IAIs patients was $100 \%$ susceptible to vancomycin, ${ }^{18}$ which however was moderately effective compared with daptomycin against Gram-positive bacterial isolates from Asia-Pacific region. ${ }^{19}$ Another in vitro study confirmed that all strains of MRSA causing severe IAIs and subsequent multiple organ failure were susceptible to vancomycin. ${ }^{20}$ However, in vivo study failed to reproduce consistent results of in vitro settings and was unable to demonstrate synergism 


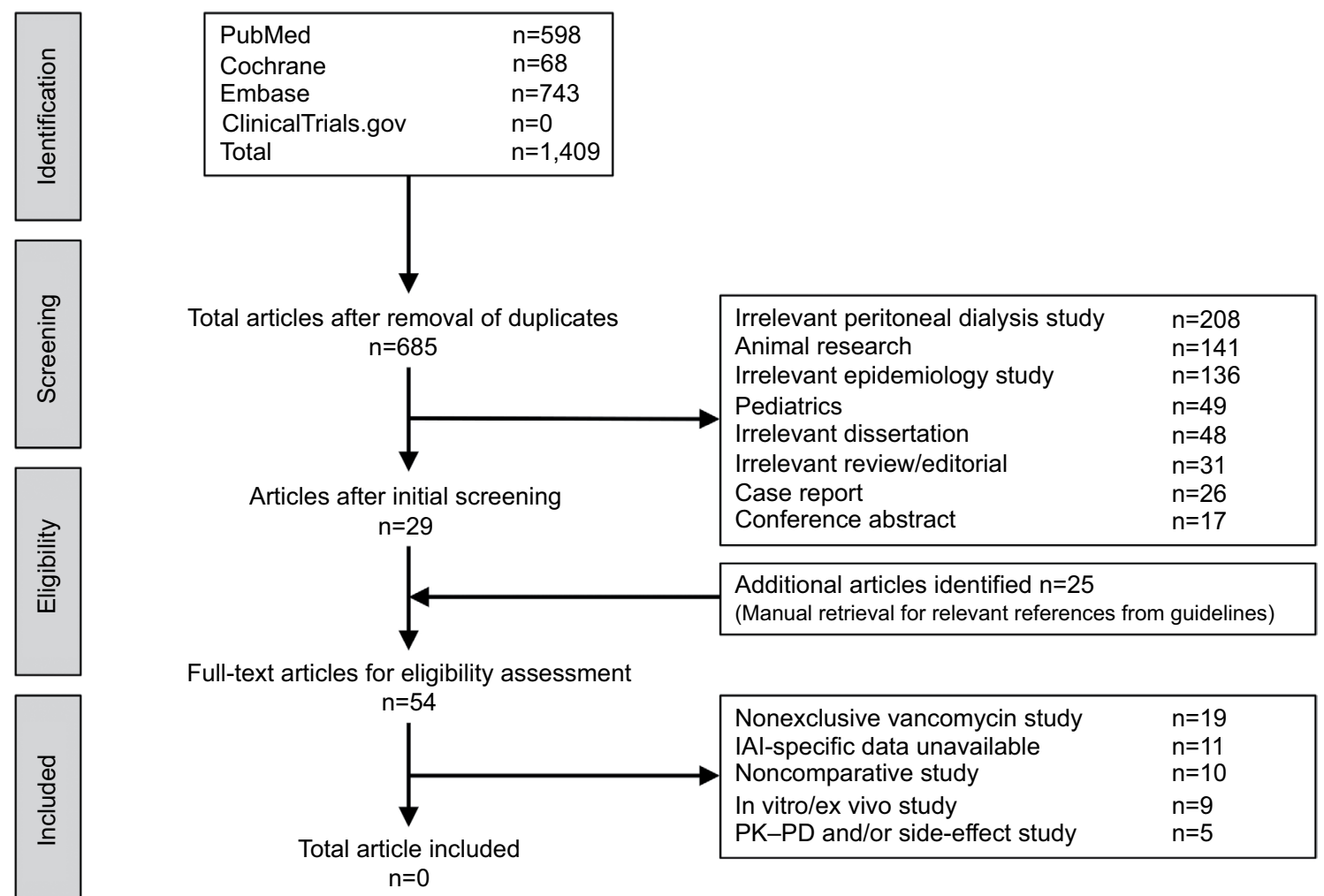

Figure I The flow chart of literature search for the exclusive use of vancomycin in intra-abdominal infections. Abbreviations: IAI, intra-abdominal infection; PD, harmacodynamics; PK, pharmacokinetics.

or antagonism effect between glycopeptides and other antibiotics. $^{21}$

By excavating the supporting references in the guidelines, we notice that current recommendations are more subjective instead of objective, which mainly originate from experts' personal experiences or from the therapeutic efficacy of vancomycin in other types of infections rather than in IAIs. Furthermore, after conducting literature search for eligible clinical studies, we discover that high-level evidence regarding the exclusive therapy of vancomycin in the treatment of IAIs is absent, and current low-level evidences are inadequate to elicit strong recommendations on the exclusive use of vancomycin in the treatment of IAIs. Our study would be helpful for rational use of vancomycin and deceleration of the emerging vancomycin resistance rates.

Compared to monotherapy, inclusion of vancomycin as part of broad-spectrum coverage failed to result in better clinical cure or reduced mortality, ${ }^{22}$ but even longer length of stay in intensive care unit. ${ }^{23}$ This was consistent with another in vivo experiment that demonstrated that glycopeptides were unable to synergize with beta-lactam antibiotics against Staphylococcus aureus-associated IAIs, ${ }^{21}$ although several single-arm studies suggested that high-dose vancomycin might help to improve the clinical efficacy against MRSA. ${ }^{24,25}$

Vancomycin was first isolated in 1953 and was approved for clinical use by Food and Drug Administration (FDA) in 1958. The patent expired in early 1980s. Since then, several generic versions from various manufacturers have been authorized by FDA for the sale of vancomycin. We assume that the lack of industry-driven clinical trials in recent 30 years contributes to the deficiency of clinical evidences in this area. We believe that future randomized clinical studies are necessary to verify current recommendations and develop new scenarios in which exclusive vancomycin is appropriate for the management of IAIs.

\section{Acknowledgments}

The authors lament the decease of the dearest friend Dr. Shifu SHA, MD, PhD, (School of Medicine, Nanjing University) who has provided significant contribution to this scientific work. The authors thank Dr Daren Juan Hsuen Low (the Agency for Science, Technology and Research, Singapore) for his language polishing and critical review of this article. Source of funding was from the National Natural Science 
Foundation of China (81602103), Natural Science Foundation of Jiangsu Province (BK20160114), Key Project of Medical Science and Technology Development Foundation of Nanjing Department of Health (YKK16114), Medical Research Program of Jiangsu Provincial Commission of Health and Family Planning (Q2017007), Distinguished Young Scholar Project of Medical Science and Technology Development Foundation of Nanjing Department of Health (JQX17005), and Wu Jieping Medical Foundation (320.2710.1817).

\section{Disclosure}

The authors report no conflicts of interest in this work.

\section{References}

1. Menichetti F, Sganga G. Definition and classification of intra-abdominal infections. J Chemother. 2009;21(Suppl 1):3-4.

2. Sartelli M. A focus on intra-abdominal infections. World J Emerg Surg. 2010;5:9.

3. Solomkin JS, Ristagno RL, das AF, et al. Source control review in clinical trials of anti-infective agents in complicated intra-abdominal infections. Clin Infect Dis. 2013;56(12):1765-1773.

4. Boneca IG, Chiosis G. Vancomycin resistance: occurrence, mechanisms and strategies to combat it. Expert Opin Ther Targets. 2003;7(3):311-328.

5. Al-Tatari H, Abdel-Haq N, Chearskul P, Asmar B. Antibiotics for treatment of resistant Gram-positive coccal infections. Indian J Pediatr. 2006;73(4):323-334.

6. Kirst HA, Thompson DG, Nicas TI. Historical yearly usage of vancomycin. Antimicrob Agents Chemother. 1998;42(5):1303-1304.

7. Centers for Disease Control and Prevention. Summary of notifiable diseases, United States 1994. MMWR Morb Mortal Wkly Rep. 1994;43(53):1-80.

8. Novak R, Henriques B, Charpentier E, Normark S, Tuomanen E. Emergence of vancomycin tolerance in Streptococcus pneumoniae. Nature. 1999;399(6736):590-593.

9. Centers for Disease Control and Prevention (CDC). Staphylococcus aureus resistant to vancomycin - United States, 2002. MMWR Morb Mortal Wkly Rep. 2002;51(26):565-567.

10. Mazuski JE, Tessier JM, May AK, et al. The Surgical Infection Society revised guidelines on the management of intra-abdominal infection. Surg Infect. 2017;18(1):1-76.

11. Sartelli M, Chichom-Mefire A, Labricciosa FM, et al. The management of intra-abdominal infections from a global perspective: 2017 WSES guidelines for management of intra-abdominal infections. World $J$ Emerg Surg. 2017;12:29.
12. Sartelli M, Weber DG, Ruppé E, et al. Antimicrobials: a global alliance for optimizing their rational use in intra-abdominal infections (AGORA). World J Emerg Surg. 2016;11:33.

13. Montravers P, Dupont H, Leone M, et al. Guidelines for management of intra-abdominal infections. Anaesth Crit Care Pain Med. 2015;34(2):117-130.

14. Kurup A, Liau KH, Ren J, et al. Antibiotic management of complicated intra-abdominal infections in adults: the Asian perspective. Ann Med Surg. 2014;3(3):85-91.

15. Solomkin JS, Mazuski JE, Bradley JS, et al. Diagnosis and management of complicated intra-abdominal infection in adults and children: guidelines by the Surgical Infection Society and the Infectious Diseases Society of America. Clin Infect Dis. 2010;50(2):133-164.

16. Montravers P, Lepape A, Dubreuil L, et al. Clinical and microbiological profiles of community-acquired and nosocomial intra-abdominal infections: results of the French prospective, observational EBIIA study. $J$ Antimicrob Chemother. 2009;63(4):785-794.

17. Augustin P, Kermarrec N, Muller-Serieys C, et al. Risk factors for multidrug resistant bacteria and optimization of empirical antibiotic therapy in postoperative peritonitis. Crit Care. 2010;14(1):R20.

18. Zhang $\mathrm{S}$, Ren $\mathrm{L}, \mathrm{LiY}$, et al. Bacteriology and drug susceptibility analysis of pus from patients with severe intra-abdominal infection induced by abdominal trauma. Exp Ther Med. 2014;7(5):1427-1431.

19. Biedenbach DJ, Bell JM, Sader HS, et al. Antimicrobial susceptibility of Gram-positive bacterial isolates from the Asia-Pacific region and an in vitro evaluation of the bactericidal activity of daptomycin, vancomycin, and teicoplanin: a SENTRY Program Report (2003-2004). Int J Antimicrob Agents. 2007;30(2):143-149.

20. Kusachi S, Sumiyama Y, Nagao J, et al. Drug susceptibility of isolates from severe postoperative intraperitoneal infections causing multiple organ failure. Surg Today. 2005;35(2):126-130.

21. Domenech A, Ribes S, Cabellos C, et al. Experimental study on the efficacy of combinations of glycopeptides and beta-lactams against Staphylococcus aureus with reduced susceptibility to glycopeptides. $J$ Antimicrob Chemother. 2005;56(4):709-716.

22. Sanders JM, Tessier JM, Sawyer RG, et al. Inclusion of vancomycin as part of broad-spectrum coverage does not improve outcomes in patients with intra-abdominal infections: a post hoc analysis. Surg Infect. 2016;17(6):694-699.

23. Petite SE, Bauer SR, Bollinger JE, Ahrens CL, Harinstein LM. Antimicrobial monotherapy versus combination therapy for the treatment of complicated intra-abdominal infections. Pharmacotherapy. 2016;36(11):1138-1144.

24. Hidayat LK, Hsu DI, Quist R, Shriner KA, Wong-Beringer A. High-dose vancomycin therapy for methicillin-resistant Staphylococcus aureus infections: efficacy and toxicity. Arch Intern Med. 2006;166(19):2138-2144.

25. Shimada K, Kobayashi H, Sunakawa K. Clinical summary of intravenous use of vancomycin hydrochloride against severe infections caused by methicillin-resistant Staphylococcus aureus. Chemotherapy. 1994;42(2):192-201.
Infection and Drug Resistance

\section{Publish your work in this journal}

Infection and Drug Resistance is an international, peer-reviewed openaccess journal that focuses on the optimal treatment of infection (bacterial, fungal and viral) and the development and institution of preventive strategies to minimize the development and spread of resistance. The journal is specifically concerned with the epidemiology of antibiotic

\section{Dovepress}

resistance and the mechanisms of resistance development and diffusion in both hospitals and the community. The manuscript management system is completely online and includes a very quick and fair peerreview system, which is all easy to use. Visit http://www.dovepress.com/ testimonials.php to read real quotes from published authors. 